\title{
Design and Analysis of a Soft Actuator for a Three-legged Soft Robot
}

\author{
Yu MIAOa, Zhi-Jiang DU ${ }^{\mathrm{b}}$ and Wei DONG ${ }^{\mathrm{c}, *}$ \\ State Key Laboratory of Robotics and System, Harbin Institute of Technology, \\ 2 Yikuang Street, Harbin, 150080, China \\ adlmiaoyu@outlook.com, b duzj01@hit.edu.cn, c dongwei@hit.edu.cn \\ ${ }^{*}$ Corresponding author
}

Keywords: Soft actuator, Soft robot, Flexible manipulator, Soft materials.

\begin{abstract}
Due to a multitude of potential applications in safe interaction with human co-workers, soft robotic manipulators have attracted attention from researchers in recent years. However, it is difficult to model a soft robot because of the flexibility of soft materials, which has limited its applications. This paper presents a modelling method for a pneumatic soft actuator based on matrix displacement method. Kinematics of a sample soft actuator is analyzed and the performance of the actuator is tested by experiments. This paper also describes the potential use of the proposed soft actuator in a three-legged soft robot.
\end{abstract}

\section{Introduction}

Compared with traditional robots, soft robots have many unique properties, e.g. good adaptability, safe interactive environment and light weight nature [1]. So they can change their shapes and sizes in a large scale. Many researchers have paid their attention to soft robotics and different types of soft robots have been developed in recent years. Using soft materials and a simple pneumatic valving system, Robert F. Shepherd et al. developed a multigait soft robot which can produce complex motion, e.g. crawling and undulation [2]. Inspired by the octopus, Cecilia Laschi et al. designed a soft robot arm actuated by cables and shape memory alloy springs and it can show the basic movements of the octopus arm, e.g. elongation, shortening and bending [3]. Based on liquid crystalline elastomer (LCE), Mikołaj Rogóz et al. reported a natural-scale soft caterpillar robot which is driven by sequential and nonreciprocal illumination, and can execute various tasks, e.g. walking up a slope and pushing objects [4]. These are good examples for soft robots with different driving modes.

Although modelling is still a big challenge in soft robotics, several recent efforts have generalized in establishing mathematical models of soft robots. Joshua Bishop-Moser et al. have investigated the kinematics of Fluid filled Fiber Reinforced Elastomeric Enclosures (FREEs) [5, 6]. Based on Timoshenko beam theory, Lukas Lindenroth et al. built the stiffness model and the kinematics model of a hydraulically-actuated soft robotics manipulator [7]. Zongkui Wang et al. used a line-segment method to model the dynamics of a soft gripper and proposed an optimization-based method to identify model parameters [8]. Unfortunately, there is still no universal way to analyze the mathematical model a soft robot.

The paper is organized as follows. The scheme of the experimental system is illustrated. Then a method based on matrix displacement to analyze the kinematics of the soft actuator is presented. Experiments are conducted to test the performance of the soft actuator. This paper also presents the potential use of the soft actuator in a three-legged soft robot and some future directions.

\section{System Design}

As presented in figure 1, the soft actuator is made of silicon rubber Ecoflex 0050, with two chambers on both sides and a jamming system in the middle. Inextensible fibers are wrapped around the outer surface of the actuator to constrain inflation, in case 'balloon effect' [9] occurs. The jamming system is a latex membrane with $2 \mathrm{~g}$ fine abrasive coffee inside. When the system is vacuumed, it would 
become stiffer than in the normal state [10]. Therefore, the stiffness of the soft actuator would increase with the jammed system. The original length of the soft actuator is $54 \mathrm{~mm}$.

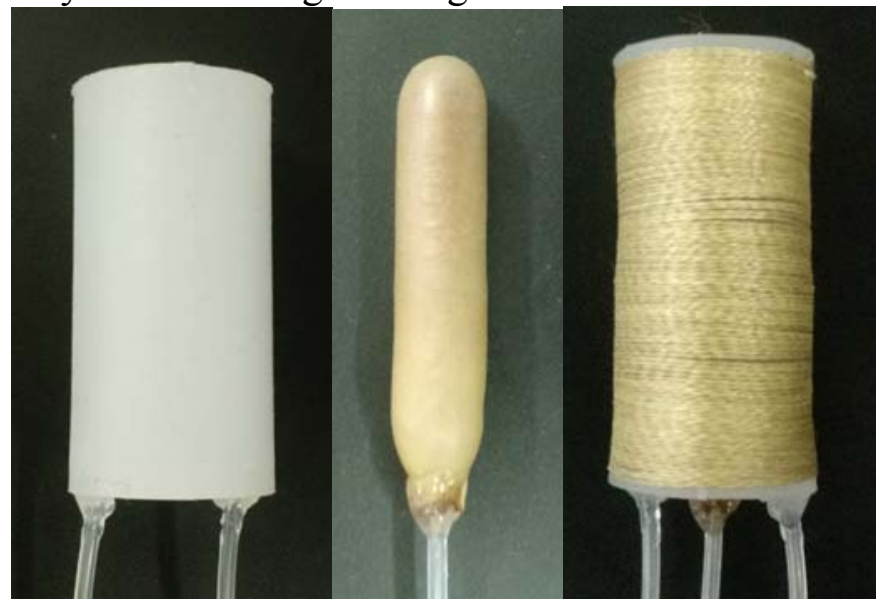

Fig. 1 Construction of the soft actuator

Figure 2 presents the setup of the experimental system. A valving system is developed to control the motion of the soft actuator. Two chambers and the jamming system are connected to the air pump and the vacuum pump via a $3 / 2$ on-off solenoid valve and a $2 / 2$ on-off solenoid valve respectively. By controlling solenoid valves, each channel can be connected to atmosphere, pressure source or hold pressure. A pressure sensor is connected to each channel to measure the pressure. Signals from pressure sensors are processed by an Arduino Mega 2560 board and then sent back to a host PC. Thus the pressure in each channel can be obtained. A user interface is developed based on Matlab GUI, through which the relay module can be controlled by the host PC. Therefore, on-off solenoid valves could be driven by the relay module to control the motion of the soft actuator.

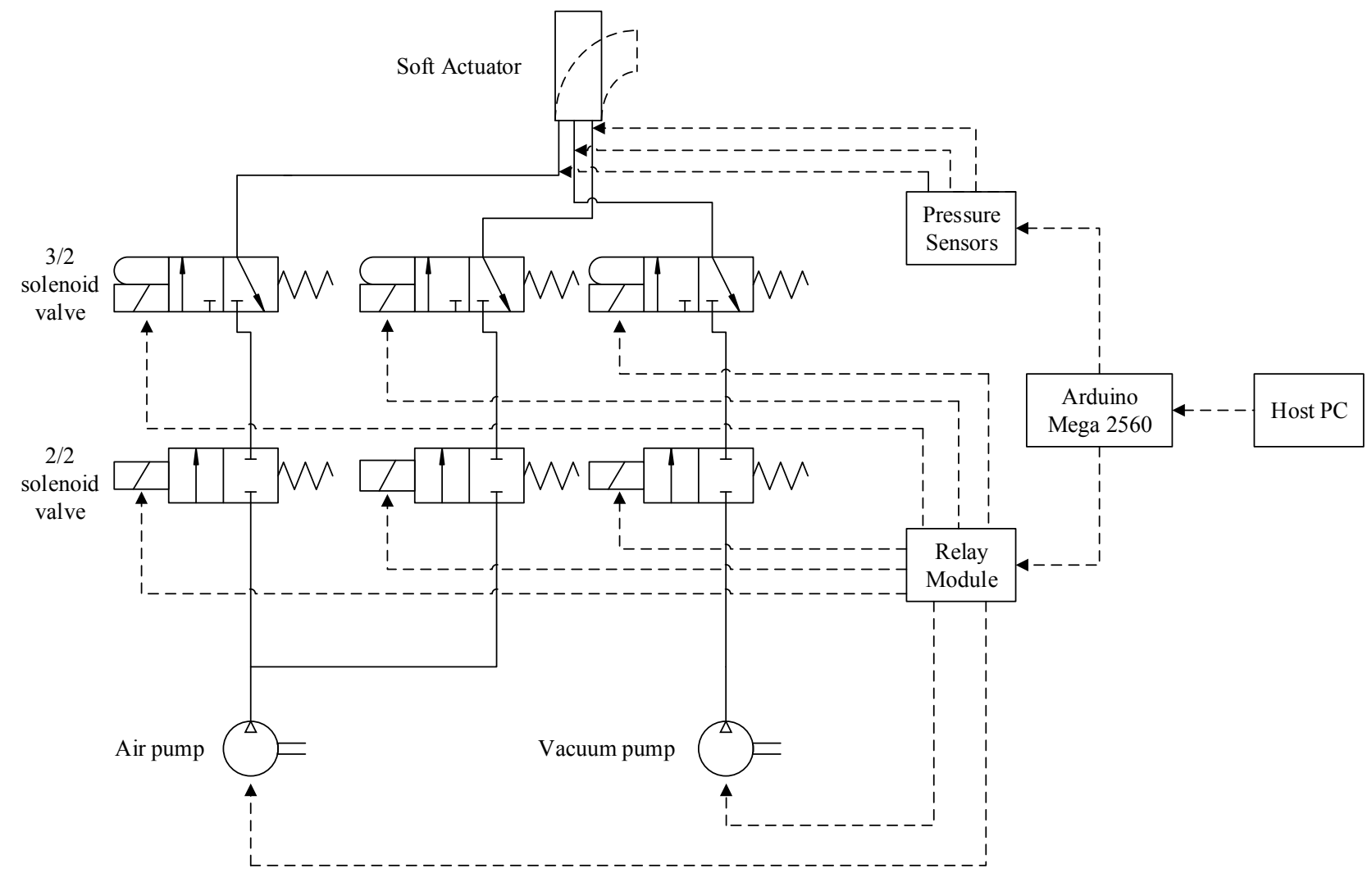

Fig. 2 Schematic diagram of the experimental system 


\section{Kinematics Modelling}

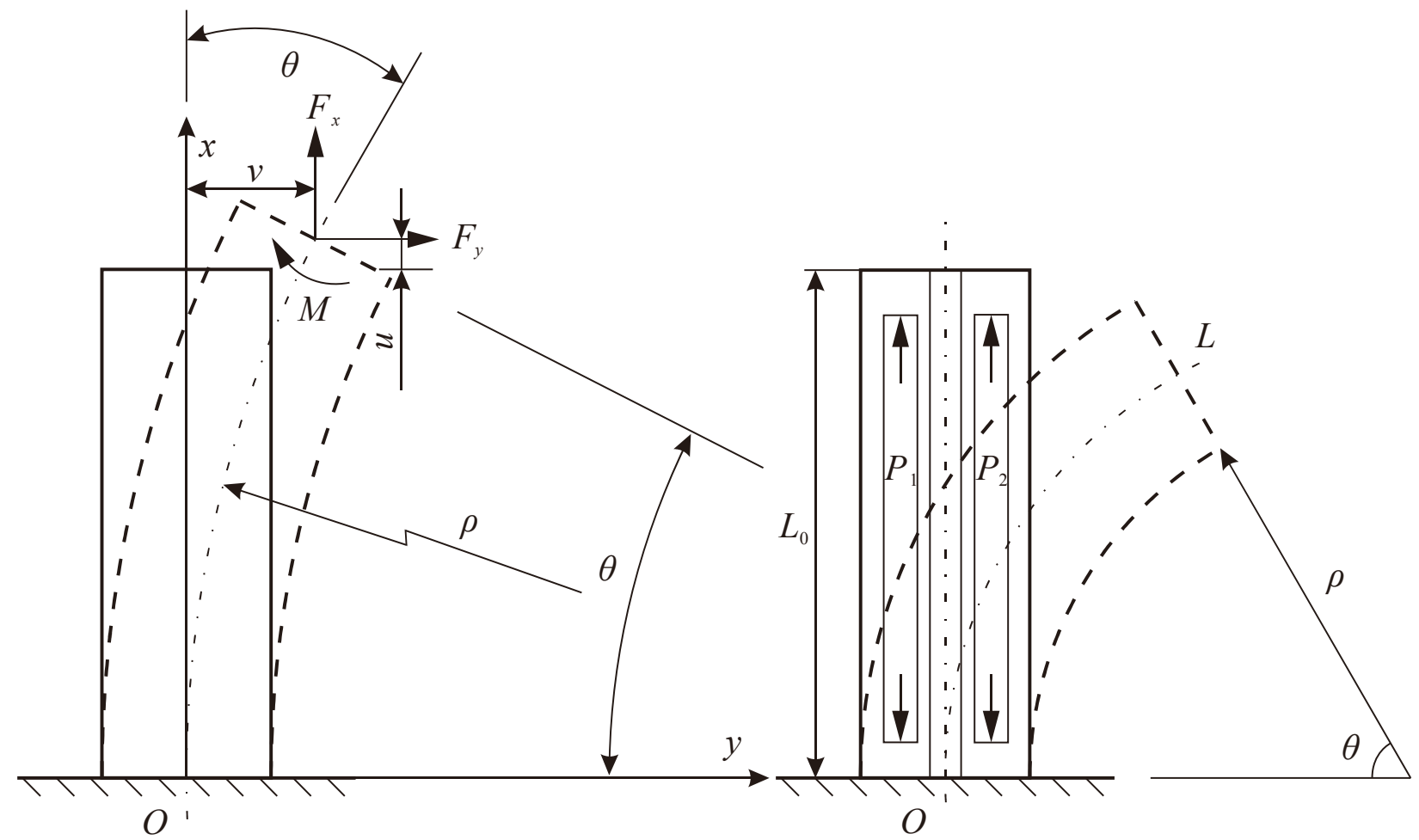

Fig. 3 Model of soft actuator

With two chambers symmetrically distributed on both sides, the soft actuator can be simplified as a cantilever beam in a plane, which is shown in figure 3. According to structural mechanics, the deflection of the actuator is related to the forces and moments applied at the tip. The stiffness equation can be expressed by

$$
\boldsymbol{F}=\boldsymbol{k} \boldsymbol{\Delta} .
$$

Where $\boldsymbol{F}$ is the external applied forces and moment and can be expressed by

$$
\boldsymbol{F}=\left(\begin{array}{lll}
F_{x} & F_{y} & M
\end{array}\right)^{\mathrm{T}} .
$$

Where $F_{x}, F_{y}$ are the forces in the $x, y$ directions respectively and $M$ is the moment applied at the tip. The position and orientation displacements can be expressed by a matrix

$$
\Delta=\left(\begin{array}{lll}
u & v & \theta
\end{array}\right)^{\mathrm{T}} .
$$

Based on structural mechanics, the stiffness matrix $\boldsymbol{k}$ can be defined as

$$
\boldsymbol{k}=\left(\begin{array}{ccc}
\frac{E A}{L_{0}} & 0 & 0 \\
0 & \frac{12 E I}{L_{0}^{3}} & -\frac{6 E I}{L_{0}^{2}} \\
0 & -\frac{6 E I}{L_{0}^{2}} & \frac{4 E I}{L_{0}}
\end{array}\right) .
$$

Where $E$ is the young modulus of the soft actuator, $I$ is the area moment of inertia and $L_{0}$ is the original length. Since matrix $\boldsymbol{k}$ is invertible, the displacements at the tip can be expressed by 


$$
\boldsymbol{\Delta}=\boldsymbol{k}^{-1} \boldsymbol{F} .
$$

Taking air pressure in the chamber into account, the forces and moment applied at the tip can be expressed by

$$
\boldsymbol{F}_{\boldsymbol{t}}=\left(F_{x}+\left(P_{1}+P_{2}\right) A_{\mathrm{c}} \quad F_{y} M+\left(P_{1}-P_{2}\right) L_{c}\right)^{\mathrm{T}} .
$$

Where $P_{1}, P_{2}$ are pressures in chamber 1 and chamber 2 respectively, $A_{\mathrm{c}}$ is the cross section of each chamber, $L_{\mathrm{c}}$ is the distance between each chamber and central axis. The displacements at the tip can be calculated by equation 5 and equation 6 .

The soft actuator can be simplified as a cantilever. When external force is applied at the tip as shown in figure 4, Young Modulus can be obtained by

$$
E=\frac{F_{v} L_{0}^{3}}{3 I v_{\max }} .
$$

Where $F_{v}$ is the external load and $v_{\max }$ is the vertical displacement of the tip.

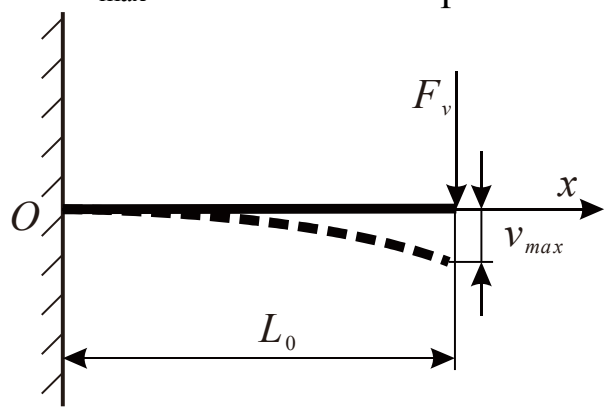

Fig. 4 Cantilever Model

\section{Experiment}

When a chamber is pressurized, the soft actuator will bend to the opposite direction. Figure 5 shows the bending range of 30,60, 90 degrees respectively. The result shows that the design method can avoid balloon effect successfully. Figure 6 shows the tip positon with the bending range of 0-120 degrees.

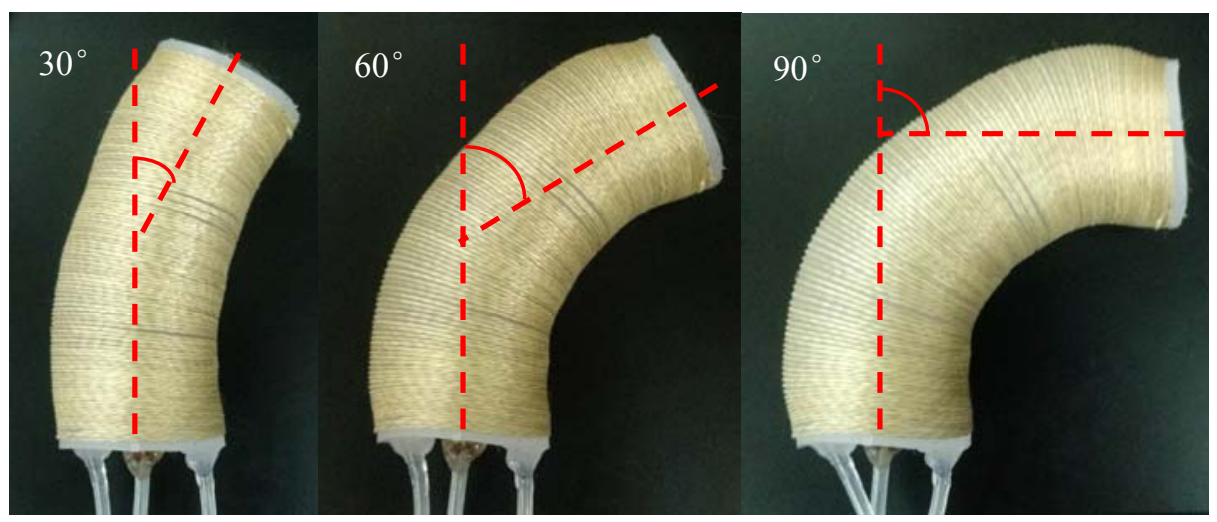

Fig. 5 Bending Status

When two chambers are pressurized at the same time, the soft actuator would extend in the central axis direction, figure 7 presents how the soft actuator elongates with the pressure range of $0-50 \mathrm{kPa}$. The experiment shows that the linear increase of the pressure in two chambers result in the linear increase of the length of the actuator. 


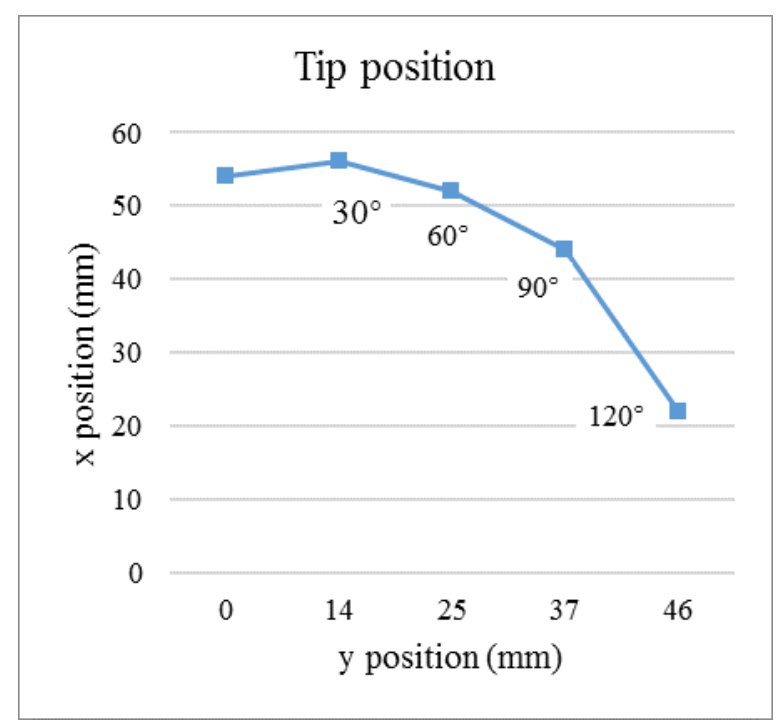

Fig. 6 Tip position

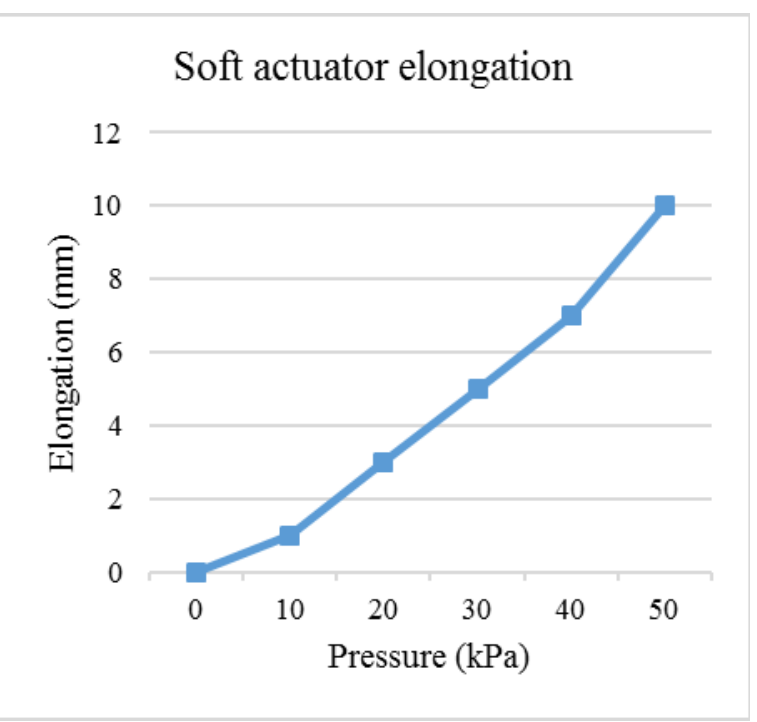

Fig. 7 Soft actuator elongation

The Young Modulus is calculated when the jamming system is in normal state and vacuumed. Figure 8 shows the test results. The jammed system can increase the Young Modulus significantly, which means the soft actuator becomes stiffer when the granular jamming is applied.

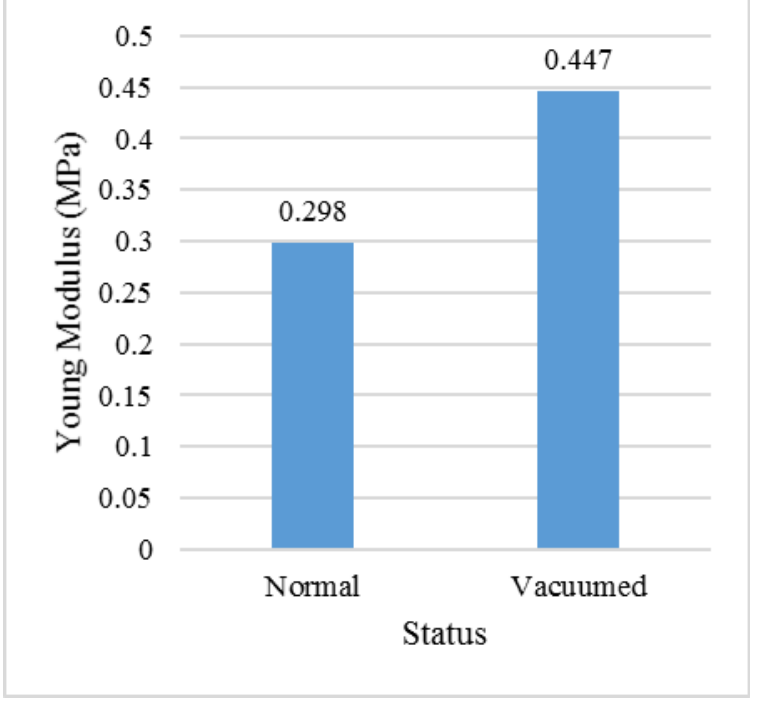

Fig. 8 Young Modulus in different status

\section{Conclusion and Future Work}

In this paper, we propose a method based on matrix displacement to analyze the kinematics of the soft actuator. Experiments show that inextensible fibers wrapped around the outer surface of the soft actuator can prevent balloon effect. Granular jamming system can increase the stiffness of the soft actuator.

In the future, the kinematics model will be refined and verified by the experiments. Based on the proposed soft actuator, a three-legged robot will be fabricated. The robot is expected to have three legs distributed in a plane, with 120 degrees between each leg. Every leg has two chambers at both sides, a jamming system in the middle and a sucker at the end. Bending occurs in the leg when chambers are pressured. Vacuumed jamming system allows high stiffness and a sucker allows the robot to 'stand' on smooth plane. By controlling air pressures in chambers, jamming systems and suckers, the soft robot is able to accomplish complex motion, such as rolling. 


\section{Acknowledgement}

This research was financially supported by the State Key Laboratory of Robotics and System, Harbin Institute of Technology under Grant No.SKLRS201501A03.

\section{References}

[1] Krishnan, G. (2014, September). Kinematics of a new class of smart actuators for soft robots based on generalized pneumatic artificial muscles. In Intelligent Robots and Systems (IROS 2014), 2014 IEEE/RSJ International Conference on (pp. 587-592). IEEE.

[2] Shepherd, R. F., Ilievski, F., Choi, W., Morin, S. A., Stokes, A. A., \& Mazzeo, A. D., et al. (2011). Multigait soft robot. Proceedings of the National Academy of Sciences of the United States of America, 108(51), 20400-3.

[3] Cecilia Laschi, Matteo Cianchetti, Barbara Mazzolai, Laura Margheri, Maurizio Follador, \& Paolo Dario. (2012). Soft robot arm inspired by the octopus. Advanced Robotics, 26(7), 709-727.

[4] Rogóż, M., Zeng, H., Xuan, C., Wiersma, D. S., \& Wasylczyk, P. (2016). Light - Driven Soft Robot Mimics Caterpillar Locomotion in Natural Scale. Advanced Optical Materials, 4(11), 1689-1694.

[5] Krishnan, G., Bishop-Moser, J., Kim, C., \& Kota, S. (2012). Evaluating Mobility Behavior of Fluid Filled Fiber-Reinforced Elastomeric Enclosures. ASME 2012 International Design Engineering Technical Conferences and Computers and Information in Engineering Conference (Vol.4, pp.1089-1099).

[6] Bishop-Moser, J., \& Kota, S. (2015). Design and modeling of generalized fiber-reinforced pneumatic soft actuators. IEEE Transactions on Robotics, 31(3), 536-545.

[7] Lindenroth, L., Back, J., Schoisengeier, A., Noh, Y., Wurdemann, H., \& Althoefer, K., et al. (2016). Stiffness-based modelling of a hydraulically-actuated soft robotics manipulator. Ieee/rsj International Conference on Intelligent Robots and Systems (pp.2458-2463). IEEE.

[8] Wang, Z., \& Hirai, S. (2017). Soft Gripper Dynamics Using a Line-Segment Model With an Optimization-Based Parameter Identification Method. IEEE Robotics and Automation Letters, 2(2), 624-631.

[9] Information on http://softroboticstoolkit.com/mmvsm/fabrication/modules

[10] Jiang, A., Secco, E. L., Würdemann, H., Nanayakkara, T., Althoefer, K., \& Dasgupta, P. (2013). Stiffness-controllable octopus-like robot arm for minimally invasive surgery. Joint Workshop on New Technologies for Computer/robot Assisted Surgery. 